\title{
S-1 plus oxaliplatin versus capecitabine plus oxaliplatin for the first-line treatment of patients with metastatic colorectal cancer: updated results from a phase 3 trial
}

Seung Tae Kim', Yong Sang Hong ${ }^{2}$, Ho Yeong Lim', Jeeyun Lee ${ }^{1}$, Tae Won Kim², Kyu-Pyo Kim², Sun Young Kim³, Ji Yeon Baek ${ }^{3}$, Jee Hyun Kim ${ }^{4}$ Keun-Wook Lee ${ }^{4}$, Ik-Joo Chung ${ }^{5}$, Sang-Hee Cho ${ }^{5}$, Kyung Hee Lee ${ }^{6}$, Sang Joon Shin, Hye Jin Kang ${ }^{8}$, Dong Bok Shin ${ }^{9}$, Jae Won Lee ${ }^{10}$, Sook Jung Jo ${ }^{11}$ and Young Suk Park ${ }^{1 *}$

\begin{abstract}
Background: We report updated progression-free survival (PFS) and overall survival (OS) data from a trial that compared capecitabine plus oxaliplatin (CapeOX) versus S-1 plus oxaliplatin (SOX) for the first-line treatment of metastatic colorectal cancer.

Methods: This trial was a randomized, two-armed, non-inferiority phase 3 comparison of CapeOX (capecitabine $1000 \mathrm{mg} / \mathrm{m} 2$ twice daily on days $1-14$ and oxaliplatin $130 \mathrm{mg} / \mathrm{m} 2$ on day 1 ) versus SOX (S-1 $40 \mathrm{mg} / \mathrm{m} 2$ twice daily on days 1-14 and oxaliplatin $130 \mathrm{mg} / \mathrm{m} 2$ on day 1). The primary end point was to show non-inferiority of SOX relative to CapeOX in terms of PFS. Thus, a follow-up exploratory analysis of PFS and OS was performed.

Results: The intention to treat (ITT) population was comprised of 340 patients (SOX arm: 168 and CapeOX arm: 172). The updated median PFS was 7.1 months $(95 \% \mathrm{Cl} 6.4-8.0)$ in the SOX group and 6.3 months $(95 \% \mathrm{Cl} 4.9-6.7)$ in the CapeOX group (hazard ratio [HR], 0.83 [0.66-1.04], $p=.10$ ). The median OS was 19.0 months ( $95 \% \mathrm{Cl} 15.3-23.0)$ in the SOX group and 18.4 months $(95 \% \mathrm{Cl} 14.1-20.7)$ in the CapeOX group (HR, 0.86 [0.68-1.08], $p=.19)$. Subgroup analyses according to principal demographic factors such as sex, age, ECOG (Eastern Cooperative Oncology Group) performance status, primary tumor location, measurability, previous adjuvant therapy, number of metastatic organs, and liver metastases showed no interaction between any of these characteristics and the treatment.
\end{abstract}

Conclusions: Updated survival analysis shows that SOX is similar to CapeOX, confirming the initial PFS analysis. Therefore, the SOX regimen could be an alternative first-line doublet chemotherapy strategy for patients with metastatic colorectal cancer.

Trial registration: NCT00677443 and May 122008

Keywords: Capecitabine, S-1, Colorectal cancers

\footnotetext{
* Correspondence: pys27hmo@skku.edu

${ }^{1}$ Department of Medicine, Samsung Medical Center, Sungkyunkwan University

School of Medicine, 81 Irwon-ro, Gangnam-gu, Seoul 135-710, Korea

Full list of author information is available at the end of the article
} 


\section{Background}

Fluoropyrimidines (5FU) have remained the most commonly prescribed agents for gastrointestinal cancer, including colorectal cancer (CRC). $5 \mathrm{FU}$ is administered as a continuous infusion by a portable pump or by an inserted chemo-port, methods that provide continuous exposure and modest improvement in efficacy. However, continuous infusion is inconvenient and unsafe [1,2]. Thus, the development of oral FU (capecitabine and S-1) has opened new possibilities for the treatment of gastrointestinal tumors, especially gastric cancers [3-5]. For the first-line treatment of patients with metastatic CRC, oxaliplatin plus either fluorouracil or capecitabine has been one of the reference doublet cytotoxic chemotherapy strategies [6,7]. S-1 is a novel oral FU consisting of a 5FU prodrug, tegafur, the dihydropyrimidine dehydrogenase inhibitor, 5-chloro-2, 4-dihydroxypyrimidine, and the orotate phosphoribosyl transferase inhibitor, potassium oxonate, which suppresses the gastrointestinal toxicity of tegafur [8]. Although several trials have shown the feasibility and efficacy of S-1 plus oxaliplatin (SOX) as an upfront chemotherapy for metastatic CRC $[9,10], \mathrm{S}-1$ and capecitabine have not been directly compared when either is combined with oxaliplatin. To address this dearth of information, we conducted our initial randomized, noninferiority phase III trial of SOX versus capecitabine plus oxaliplatin (CapeOX) for the first-line treatment of patients with metastatic colorectal cancer [11]. We found the S-1 group to have nearly 2 months longer PFS than the capecitabine group, suggesting that the SOX regimen could be an alternative first-line doublet chemotherapy strategy for patients with metastatic CRC. However, which particular S-1s can be used as substitutes for capecitabine may be controversial in CRC. Thus, we intend to update the overall survival (OS) and progression free survival (PFS) results, and we intend to conduct exploratory analyses to determine whether the effect of S-1 on these end points appears to vary in selected patient groups.

\section{Methods}

\section{Study design}

This was a randomized, open-label, multicenter phase 3 study [11]. The institutional review boards of all participating institutions approved the study protocol. Written, informed consent was required for participation.

To be eligible, patients with metastatic colorectal cancer were required to have histologically confirmed adenocarcinoma, measurable or assessable lesions, an Eastern Cooperative Oncology Group (ECOG) performance status (PS) of 0-2, no previous chemotherapy or immunotherapy in a metastatic setting, adequate hematological, hepatic, and renal function, and be 18 years of age or older. Adjuvant chemotherapy or radiotherapy was permitted if it had been completed at least 6 months before enrollment. We randomly assigned eligible patients to either CapeOX or SOX in a one-to-one ratio. Randomisation was done centrally with a computergenerated sequence and a permutation block technique that ensured equal distribution of patients on the basis of primary tumor site (colon vs. rectum), history of previous adjuvant or neoadjuvant treatment, and the presence of measurable lesions.

\section{Procedures}

All treatment cycles were administered every three weeks. We administered oral S-1 $(40 \mathrm{mg} / \mathrm{m} 2)$ twice a day on days $1-14$, oral capecitabine $(1000 \mathrm{mg} / \mathrm{m} 2)$ twice a day on days $1-14$, and oxaliplatin $(130 \mathrm{mg} / \mathrm{m} 2)$ on day 1 as a 2-h intravenous infusion. As many as nine cycles of oxaliplatin-containing chemotherapy were provided, except in instances of disease progression, unacceptable toxicity, or patient refusal. Maintenance chemotherapy with S-1 or capecitabine was permitted after discontinuation of oxaliplatin. Treatment responses were assessed every three cycles ( 9 weeks) during study treatments or sooner if needed for documentation of disease progression. Objective tumor responses were independently reviewed according to the Response Evaluation Criteria In Solid Tumors (RECIST; version 1.0).

\section{Statistical analyses}

For the primary efficacy analysis, in which we aimed to assess the non-inferiority of SOX to CapeOX in terms of PFS (time to progression or death), we assessed all patients allocated to the treatment group (intention to treat population), and we also did a per-protocol analysis in those who received protocol treatments without major violations. PFS at 15 months in both groups was assumed to be $38 \%$, and the low non-inferiority limit was set as $-13 \%$, corresponding to a HR of 1.43 . On the basis of these conditions, 192 events were needed for a one-sided type I error of $5 \%$ and a power of $80 \%$. Assuming a $10 \%$ loss, we needed 344 patients. Previous reports were made at the August 31, 2011 cut-off date for data collection. These updated data were collected at the cut-off date of December 24, 2013. The survival data were assessed with the Kaplan-Meier method. We estimated the hazard ratio (HR) and corresponding 95\% CI using the Cox proportional hazard regression model. Also, we analyzed post-progression survival (PPS; the duration of survival after disease progression to study medication) among two groups.

\section{Results}

\section{Patients}

Between May 14, 2008 and September 23, 2009, we enrolled 348 patients from 11 institutions. We randomly assigned 340 patients who met the eligibility criteria to 


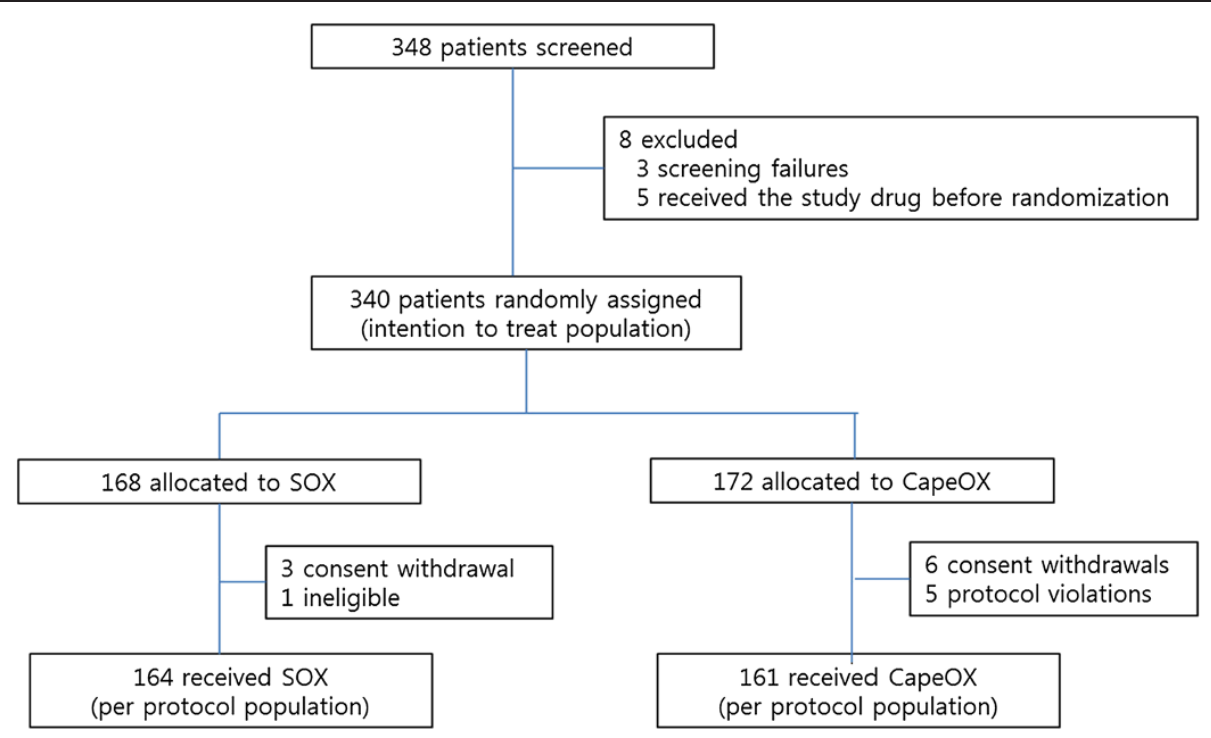

Figure 1 CONSORT diagram.

treatment (ITT). Of the 340 patients, 168 were assigned to the SOX group, and 172 were assigned to the CapeOX group (Figure 1). This updated analysis used data collected by December 24, 2013, by which time, 301 (SOX group: 150, CapeOX group: 151) PFS events, and 279 (134; SOX group, 145; CapeOX group) OS events had occurred. In the previous analysis, 207 (98; SOX group, 109; CapeOX group) PFS events, and 179 (84; SOX group, 95; CapeOX group) OS events had occurred. Of note, the baseline characteristics were much the same between the two groups (Table 1).

\section{Progression-free survival (PFS) and post-progression survival (PPS)}

In the SOX group with media 17.91 follow-up, the median PFS was 7.1 months (6.4-8.1), and the corresponding value in the CapeOX group with median 16.41 follow-up was 6.3 months (4.9-6.7, intention to treat [ITT] population) (Table 2 and Figure 2). The HR comparing PFS between the two groups was $0.83(95 \%$ CI $0.66-1.04, p=.10)$. For the ITT population, the median post-progression survival (PPS) was 9.3 months in the SOX group compared to 9.5 months in the CapeOX group, with a corresponding HR of 0.97 (95\% CI 0.76-1.23, $p=.81$ ) (Table 2 and Figure 2).

\section{Overall survival (OS)}

The OS data in the ITT population as of December 24, 2013 are shown in Table 2. The corresponding KaplanMeier curves for OS are shown in Figure 3.

For the ITT population, median OS was 19.0 months in the SOX group compared to 18.5 months in the CapeOX group, with a corresponding HR of 0.86 (95\% CI 0.68-1.08).
Table 1 Baseline characteristics (intention to treat population)

SOX $(n=168) \quad$ CapeOX $(n=172)$

\begin{tabular}{|c|c|c|}
\hline \multicolumn{3}{|l|}{ Primary site } \\
\hline Colon & $109(65 \%)$ & $108(63 \%)$ \\
\hline Rectum & $59(35 \%)$ & $64(37 \%)$ \\
\hline \multicolumn{3}{|l|}{ Sex } \\
\hline Male & $109(65 \%)$ & $102(59 \%)$ \\
\hline Female & 59 (35\%) & 70 (41\%) \\
\hline \multicolumn{3}{|l|}{ Age } \\
\hline$\leq 65$ years & $121(72 \%)$ & $126(73 \%)$ \\
\hline$>65$ years & $47(28 \%)$ & $46(27 \%)$ \\
\hline \multicolumn{3}{|l|}{ ECOG performance status } \\
\hline $0-1$ & $164(98 \%)$ & $168(98 \%)$ \\
\hline 2 & $4(2 \%)$ & $4(2 \%)$ \\
\hline \multicolumn{3}{|c|}{ Previous (neo)adjuvant therapy } \\
\hline Yes & $37(22 \%)$ & $38(22 \%)$ \\
\hline No & $131(78 \%)$ & $134(78 \%)$ \\
\hline \multicolumn{3}{|l|}{ Site of metastasis } \\
\hline Liver metastasis & $105(63 \%)$ & $111(65 \%)$ \\
\hline Non-liver metastasis & $63(37 \%)$ & $61(35 \%)$ \\
\hline \multicolumn{3}{|l|}{ Number of metastatic organs } \\
\hline One organ & 65 (39\%) & $49(29 \%)$ \\
\hline Two organs & $61(36 \%)$ & $70(41 \%)$ \\
\hline Three or more organs & $42(25 \%)$ & $53(31 \%)$ \\
\hline \multicolumn{3}{|l|}{ Measurability } \\
\hline Measurable lesions & $155(92 \%)$ & 155 (90\%) \\
\hline Assessable lesions only & $13(8 \%)$ & $17(10 \%)$ \\
\hline
\end{tabular}


Table 2 Survival outcomes

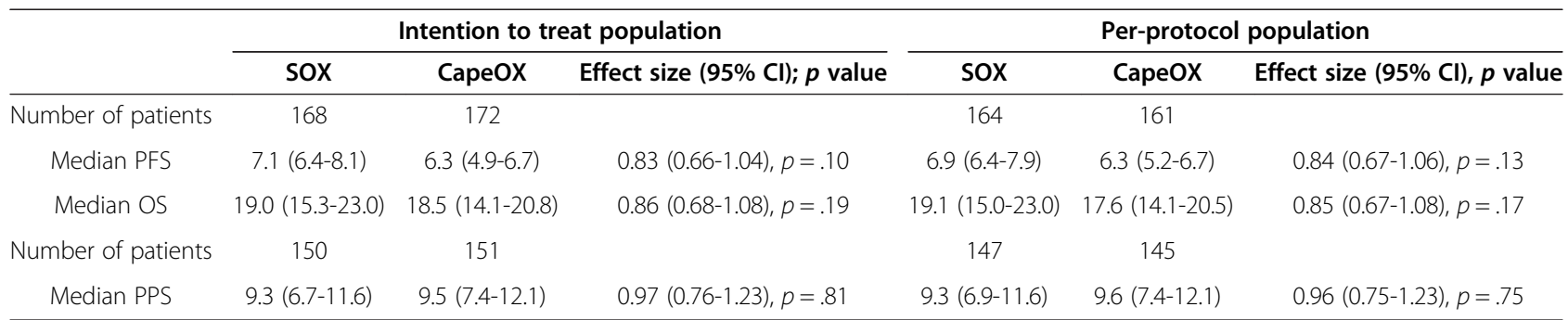

Data are represented as $\mathrm{n}(\%)$, time in months $(95 \% \mathrm{Cl})$ or effect size $(95 \% \mathrm{Cl})$. SOX, S-1 plus oxaliplatin; CapeOX, Capecitabine plus oxaliplatin; PFS, progression-free survival; PPS, post-progression survival, OS = overall survival.

\section{Subgroup analysis}

OS and PFS in assigned patients were analyzed according to sex, age, ECOG PS, primary tumor site, previous adjuvant therapy, measurability, number of metastatic organs, and liver metastasis. There was no interaction between the treatment and any of these factors (Figure 4).

\section{Discussion}

The primary analysis of this study showed that SOX is non-inferior to CapeOX in terms of PFS, OS, and response rate as a first-line chemotherapy in patients with metastatic colorectal cancer (CRC) [11]. To the best of our knowledge, this study is the first large, phase III clinical trial that directly compares SOX with CapeOX in metastatic CRC. This updated analysis also demonstrates that SOX and CapeOX have statistically similar PFS and OS, confirming the conclusion reached in our previous publication.

In this updated analysis, patients in the CapeOX group had a PFS of 6.3 months and an OS of 18.5 months, which are similar values to those reported in previous trials $[6,12-14]$. Likewise, the SOX group in our analysis showed favorable results, which are similar to values recorded for CapeOX or FU plus oxaliplatin plus Leucovorin (FOLFOX) treatment in previous studies [12-14].
Based on our updated analysis with a long-term followup, no significant differences were observed in PFS and OS between the SOX and CapeOX groups, which is consistent with previous gastrointestinal cancer studies [15-18]. In addition, a meta-analysis by Zhang et al. reported that both the S-1 and capecitabine-based regimens were equally active and well tolerated in patients with gastric cancer and CRC [19].

In this updated analysis, we also investigated the postprogression survival (PPS) for both treatment groups. In cancer treatment, the goal is to prolong survival and/or to improve the patient's quality of life. In the era of multiple lines of chemotherapy, therapeutic options have been expanded, especially in CRC. In breast cancer, lung cancer, and CRC, OS becomes more associated with PPS than PFS [20-23]. In CRC, the association of PPS with OS may be due to the increasing number of active compounds available, or to the available second or third-line therapies. In this analysis, there was no significant difference in PPS between the SOX and CapeOX groups (Table 2). These findings suggest that effective treatments, equal in quality, were provided to both groups after the failure of the first-line therapy. The dose of S-1 in this study was $80 \mathrm{mg} / \mathrm{m} 2$ per day, which is higher

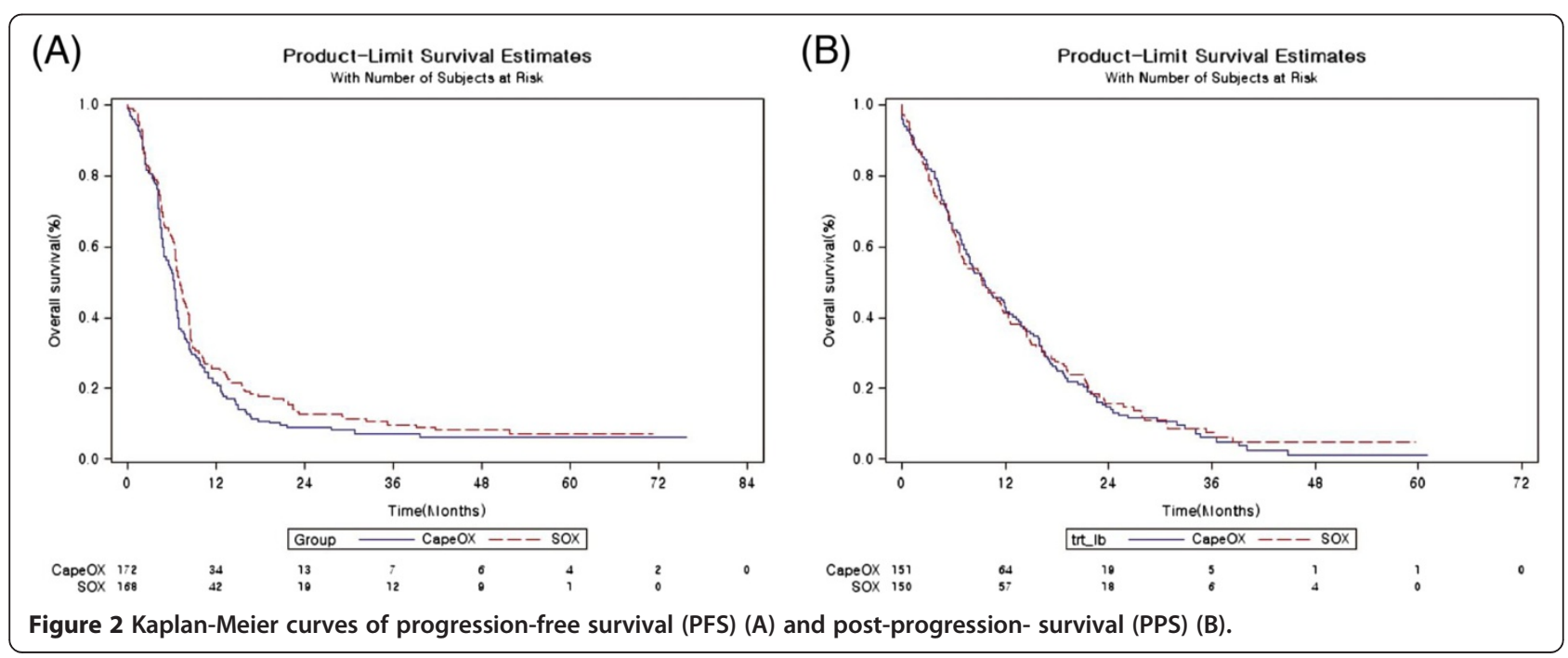




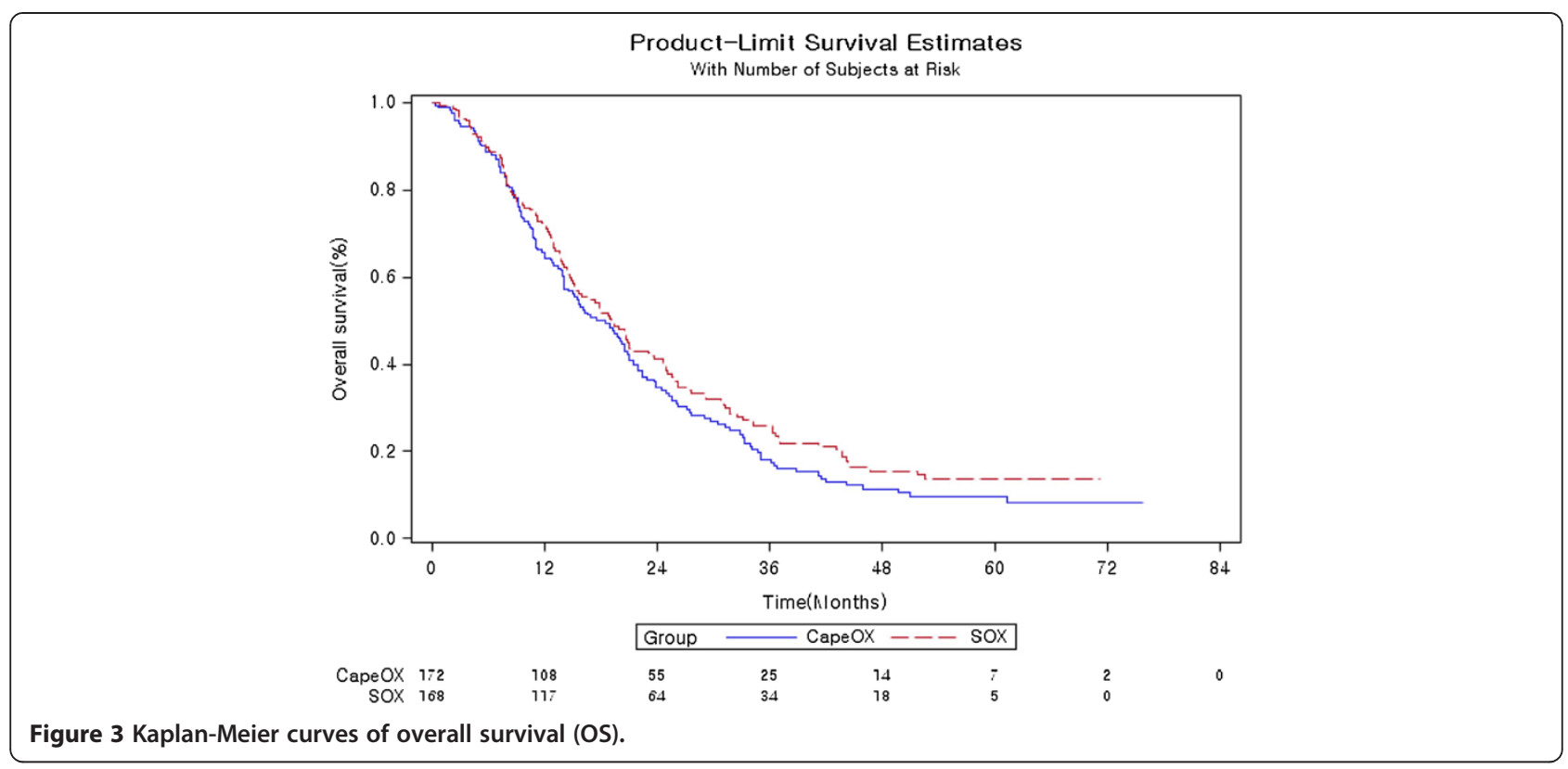

than doses used in the reference trial that combined S-1 with oxaliplatin. As mentioned in a previously published report, this dose finding explains the high frequency of adverse events in SOX-treated patients. However, the high frequency of adverse events in the SOX group might not affect subsequent treatment strategies.
In patients with metastatic CRC, the addition of bevacizumab to the first-line chemotherapy has improved survival. Previous studies have shown that bevacizumab plus FOLFOX, FOLFIRI (leucovorin, fluorouracil, and irinotecan), or CapeOX improves PFS [12,14,24-26]. However, the role of bevacizumab with SOX has not

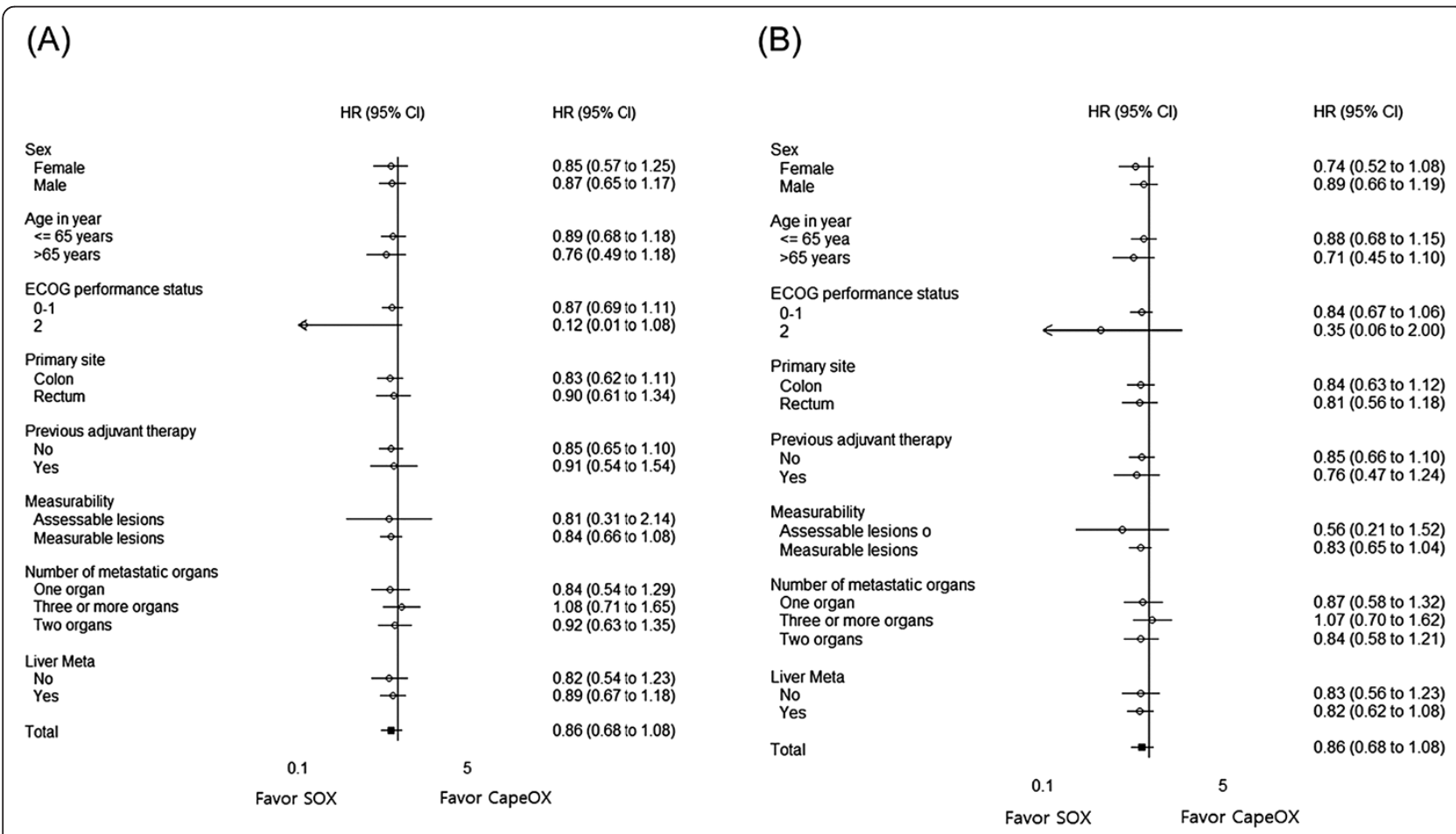

Figure 4 Subgroup analysis of overall survival (A) and progression-free survival (B) for the intention to treat population. 
been established. Recently, Yamada et al. showed that bevacizumab plus SOX was non-inferior to bevacizumab plus FOLFOX as a first-line treatment for metastatic CRC with respect to PFS [27]. Thus, bevacizumab plus SOX might be a possible option as a standard first-line treatment, although S-1 and capecitabine have not been directly compared when either is combined with bevacizumab and oxaliplatin.

Our updated analysis confirms that a combination of S-1 and oxaliplatin can be considered as an alternative doublet chemotherapy strategy to CapeOX. Further investigation is needed to explore its potential when used together with other targeted agents or as adjuvant chemotherapy.

\section{Conclusion}

Updated survival analysis shows that SOX is similar to CapeOX, confirming the initial PFS analysis. Therefore, the SOX regimen could be an alternative first-line doublet chemotherapy strategy for patients with metastatic colorectal cancer.

\section{Ethics statement}

The Ethics Committee at Samsung Medical Center approved the study in accordance with the Declaration of Helsinki. All individuals gave written informed consent for participation in the study.

\section{Competing interests}

The authors declare that they have no competing interests.

\section{Authors' contributions}

Conception and design were carried out by TWK, DBS, JWL and YSP. Development of methodology was conducted by SJJ, JWL, STK, and HYL. STK, KPK, SYK, JYB, JHK, KWL, IJC, SHC, KHL, SJS, HJK and YSP participated in Acquisition of data. Analysis and interpretation of data were conducted by SJ, STK, and JL. Writing, review and/or revision of manuscript were carried out by STK, YSH, JL, and YSP. KPK, SYK, JYB, JHK, KWL, IJC, SHC, KHL, SJS, and HJK participated in administrative, technical or material support. Study supervision was conducted by YSP. All authors read and approved the final manuscript.

\section{Acknowledgements}

This study was partly supported by a grant from the Korea Healthcare technology R\&D Project, Ministry for Health, Welfare \& Family Affairs, Republic of Korea (Grant Number.; A102065). The oxaliplatin and S-1 were provided by Jeil Pharmaceutical (Seoul, South Korea). These mentions was already revealed at previous article (Lancet Oncol 2012;13:1125). This updated-study didn't need additional any fund.

\footnotetext{
Author details

${ }^{1}$ Department of Medicine, Samsung Medical Center, Sungkyunkwan University School of Medicine, 81 Irwon-ro, Gangnam-gu, Seoul 135-710, Korea. ${ }^{2}$ Department of Oncology, Asan Medical Center, University of Ulsan College of Medicine, Seoul, Korea. ${ }^{3}$ Centre for Colorectal Cancer, National Cancer Center, Goyang, Korea. ${ }^{4}$ Department of Internal Medicine, Seoul National University Bundang Hospital, Seoul National University College of Medicine, Seongnam, Korea. ${ }^{5}$ Department of Internal Medicine, Chonnam National University Hwasun Hospital, Chonnam National University Medical School, Jeollanamdo, Korea. ${ }^{6}$ Department of Internal Medicine, Yeungnam University Hospital, Yeungnam University College of Medicine, Daegu, Korea. ${ }^{7}$ Department of Internal Medicine, Cancer Metastasis Research Centre, Yonsei University College of Medicine, Seoul, Korea. ${ }^{8}$ Department of Internal Medicine, Korea Cancer Centre Hospital, Korea Institute of Radiological and Medical Sciences, Seoul, Korea. ${ }^{9}$ Department of Internal Medicine, Gachon University Gil Hospital, Incheon, Korea.
}

${ }^{10}$ Department of Statistics, Korea University, Seoul, Korea. ${ }^{11}$ Biostatistics, ICON Clinical Research, Seoul, Korea.

Received: 27 May 2014 Accepted: 18 November 2014 Published: 26 November 2014

\section{References}

1. Schoffski P: The modulated oral fluoropyrimidine prodrug S-1, and its use in gastrointestinal cancer and other solid tumors. Anticancer Drugs 2004 15:85-106.

2. Meta-analysis Group In Cancer: Efficacy of intravenous continuous infusion of fluorouracil compared with bolus administration in advanced colorectal cancer. Meta-analysis group in cancer. J Clin Oncol 1998, 16:301-308.

3. Cascinu S: Oral treatment for gastric cancer: new choices, better choices? Lancet Oncol 2008, 9:188-189.

4. Cervantes A, Roda D, Tarazona N, Rosello S, Perez-Fidalgo JA: Current questions for the treatment of advanced gastric cancer. Cancer Treat Rev 2013, 39:60-67.

5. Pieters A, Laurent S, Dero I, Van Damme N, Peeters M: The role of oral fluoropyrimidines in the treatment of advanced gastric cancer. Acta Gastroenterol Belg 2008, 71:361-366.

6. Cassidy J, Tabernero J, Twelves C, Brunet R, Butts C, Conroy T, Debraud F, Figer A, Grossmann J, Sawada N, Schoffski P, Sobrero A, Van Cutsem E, Diaz-Rubio E: XELOX (capecitabine plus oxaliplatin): active first-line therapy for patients with metastatic colorectal cancer. J Clin Oncol 2004, 22:2084-2091.

7. de Gramont A, Figer A, Seymour M, Homerin M, Hmissi A, Cassidy J, Boni C, Cortes-Funes H, Cervantes A, Freyer G, Papamichael D, Le Bail N, Louvet C, Hendler D, de Braud F, Wilson C, Morvan F, Bonetti A: Leucovorin and fluorouracil with or without oxaliplatin as first-line treatment in advanced colorectal cancer. J Clin Oncol 2000, 18:2938-2947.

8. Maehara Y: S-1 in gastric cancer: a comprehensive review. Gastric Cancer 2003, 6(Suppl 1):2-8.

9. Yamada Y, Tahara M, Miya T, Satoh T, Shirao K, Shimada Y, Ohtsu A, Sasaki Y, Tanigawara Y: Phase I/II study of oxaliplatin with oral S-1 as first-line therapy for patients with metastatic colorectal cancer. Br J Cancer 2008, 98:1034-1038

10. Zang DY, Lee BH, Park HC, Song HH, Kim HJ, Jung JY, Kim JH, Kim HY, Kwon $\mathrm{JH}$, Hwang SW, Park SR, Park CH, Kim KO, Kim MJ, Jang KM: Phase II study with oxaliplatin and S-1 for patients with metastatic colorectal cancer. Ann Oncol 2009, 20:892-896.

11. Hong YS, Park YS, Lim HY, Lee J, Kim TW, Kim KP, Kim SY, Baek JY, Kim JH, Lee KW, Chung IJ, Cho SH, Lee KH, Shin SJ, Kang HJ, Shin DB, Jo SJ, Lee JW: S-1 plus oxaliplatin versus capecitabine plus oxaliplatin for first-line treatment of patients with metastatic colorectal cancer: a randomised, non-inferiority phase 3 trial. Lancet Oncol 2012, 13:1125-1132.

12. Cassidy J, Clarke S, Diaz-Rubio E, Scheithauer W, Figer A, Wong R, Koski S, Rittweger K, Gilberg F, Saltz L: XELOX vs FOLFOX-4 as first-line therapy for metastatic colorectal cancer: N016966 updated results. Br J Cancer 2011, 105:58-64.

13. Rothenberg ML, Cox JV, Butts C, Navarro M, Bang YJ, Goel R, Gollins S, Siu LL, Laguerre S, Cunningham D: Capecitabine plus oxaliplatin (XELOX) versus 5 -fluorouracil/folinic acid plus oxaliplatin (FOLFOX-4) as secondline therapy in metastatic colorectal cancer: a randomized phase III noninferiority study. Ann Oncol 2008, 19:1720-1726.

14. Saltz LB, Clarke S, Diaz-Rubio E, Scheithauer W, Figer A, Wong R, Koski S, Lichinitser M, Yang TS, Rivera F, Couture F, Sirzen F, Cassidy J: Bevacizumab in combination with oxaliplatin-based chemotherapy as first-line therapy in metastatic colorectal cancer: a randomized phase III study. J Clin Oncol 2008, 26:2013-2019.

15. Lee JL, Kang YK, Kang HJ, Lee KH, Zang DY, Ryoo BY, Kim JG, Park SR, Kang WK, Shin DB, Ryu MH, Chang HM, Kim TW, Baek JH, Min YJ: A randomised multicentre phase II trial of capecitabine vs S-1 as first-line treatment in elderly patients with metastatic or recurrent unresectable gastric cancer. Br J Cancer 2008, 99:584-590.

16. Kim GM, Jeung HC, Rha SY, Kim HS, Jung I, Nam BH, Lee KH, Chung HC: A randomized phase II trial of S-1-oxaliplatin versus capecitabineoxaliplatin in advanced gastric cancer. Eur J Cancer 2012, 48:518-526.

17. Seol YM, Song MK, Choi YJ, Kim GH, Shin HJ, Song GA, Chung JS, Cho GJ: Oral fluoropyrimidines (capecitabine or S-1) and cisplatin as first line treatment in elderly patients with advanced gastric cancer: a retrospective study. Jpn J Clin Oncol 2009, 39:43-48. 
18. Shitara K, Sawaki A, Matsuo K, Kondo C, Takahari D, Ura T, Tajika M, Niwa Y, Muro K: A retrospective comparison of S-1 plus cisplatin and capecitabine plus cisplatin for patients with advanced or recurrent gastric cancer. Int $J$ Clin Oncol 2013, 18:539-546.

19. Zhang X, Cao C, Zhang Q, Chen Y, Gu D, Shen Y, Gong Y, Chen J, Tang C: Comparison of the efficacy and safety of S-1-based and capecitabine-based regimens in gastrointestinal cancer: a meta-analysis. PLoS One 2014, 9:e84230.

20. Petrelli $\mathrm{F}$, Barni $\mathrm{S}$ : Correlation of progression-free and post-progression survival with overall survival in advanced colorectal cancer. Ann Oncol 2013, 24:186-192.

21. Zietemann VD, Schuster T, Duell TH: Post-study therapy as a source of confounding in survival analysis of first-line studies in patients with advanced non-small-cell lung cancer. J Thorac Dis 2011, 3:88-98.

22. Hotta K, Kiura K, Fujiwara Y, Takigawa N, Hisamoto A, Ichihara E, Tabata M, Tanimoto M: Role of survival post-progression in phase III trials of systemic chemotherapy in advanced non-small-cell lung cancer: a systematic review. PLoS One 2011, 6:e26646.

23. Broglio KR, Berry DA: Detecting an overall survival benefit that is derived from progression-free survival. J Natl Cancer Inst 2009, 101:1642-1649.

24. Cassidy J, Clarke S, Diaz-Rubio E, Scheithauer W, Figer A, Wong R, Koski S, Lichinitser M, Yang TS, Rivera F, Couture F, Sirzen F, Saltz L: Randomized phase III study of capecitabine plus oxaliplatin compared with fluorouracil/folinic acid plus oxaliplatin as first-line therapy for metastatic colorectal cancer. J Clin Oncol 2008, 26:2006-2012.

25. Goldberg RM, Sargent DJ, Morton RF, Fuchs CS, Ramanathan RK, Williamson SK, Findlay BP, Pitot HC, Alberts SR: A randomized controlled trial of fluorouracil plus leucovorin, irinotecan, and oxaliplatin combinations in patients with previously untreated metastatic colorectal cancer. J Clin Oncol 2004, 22:23-30.

26. Tournigand C, Andre T, Achille E, Lledo G, Flesh M, Mery-Mignard D, Quinaux E, Couteau C, Buyse M, Ganem G, Landi B, Colin P, Louvet C, de Gramont A: FOLFIRI followed by FOLFOX6 or the reverse sequence in advanced colorectal cancer: a randomized GERCOR study. J Clin Oncol 2004, 22:229-237.

27. Yamada Y, Takahari D, Matsumoto H, Baba H, Nakamura M, Yoshida K, Yoshida M, Iwamoto S, Shimada K, Komatsu Y, Sasaki Y, Satoh T, Takahashi K, Mishima H, Muro K, Watanabe M, Sakata Y, Morita S, Shimada Y, Sugihara K: Leucovorin, fluorouracil, and oxaliplatin plus bevacizumab versus S-1 and oxaliplatin plus bevacizumab in patients with metastatic colorectal cancer (SOFT): an open-label, non-inferiority, randomised phase 3 trial. Lancet Oncol 2013, 14:1278-1286.

doi:10.1186/1471-2407-14-883

Cite this article as: Kim et al:: S-1 plus oxaliplatin versus capecitabine plus oxaliplatin for the first-line treatment of patients with metastatic colorectal cancer: updated results from a phase 3 trial. BMC Cancer 2014 14:883

\section{Submit your next manuscript to BioMed Central and take full advantage of:}

- Convenient online submission

- Thorough peer review

- No space constraints or color figure charges

- Immediate publication on acceptance

- Inclusion in PubMed, CAS, Scopus and Google Scholar

- Research which is freely available for redistribution 